\title{
In Situ Measurements of Elastic Constants In Liquid Crystalline Droplets
}

\author{
Vladimir Yu. Rudyak ( $\nabla$ vurdizm@gmail.com ) \\ Lomonosov Moscow State University \\ Mikhail N. Krakhalev \\ Federal Research Center KSC SB RAS \\ Anna P. Gardymova \\ Siberian Federal University \\ Abylgazy S. Abdullaev \\ Federal Research Center KSC SB RAS \\ Andrey A. Alekseev \\ Lomonosov Moscow State University \\ Victor Ya. Zyryanov \\ Federal Research Center KSC SB RAS
}

\section{Research Article}

Keywords:

Posted Date: March 7th, 2022

DOI: https://doi.org/10.21203/rs.3.rs-1365822/v1

License: (c) (1) This work is licensed under a Creative Commons Attribution 4.0 International License.

Read Full License 


\title{
In situ measurements of elastic constants in liquid crystalline droplets
}

\author{
Vladimir Yu. Rudyak ${ }^{1, *}$, Mikhail N. Krakhalev ${ }^{2,3}$, Anna P. Gardymova ${ }^{3}$, Abylgazy S. \\ Abdullaev $^{2,3}$, Andrey A. Alekseev ${ }^{1}$, and Victor Ya. Zyryanov ${ }^{2}$ \\ ${ }^{1}$ Faculty of Physics, Lomonosov Moscow State University, 119991 Moscow, Russia \\ ${ }^{2}$ Kirensky Institute of Physics, Federal Research Center KSC SB RAS, 660036 Krasnoyarsk, Russia \\ ${ }^{3}$ Institute of Engineering Physics and Radio Electronics, Siberian Federal University, 660041 Krasnoyarsk, Russia \\ *vurdizm@gmail.com
}

\begin{abstract}
In this work, we investigated the behavior of cholesteric droplets with homeotropic boundary conditions experimentally and by computer simulations. Small droplets forming twisted radial structures were studied. We obtained two different paths of structural transformations under electric field in such droplets. The choice between these paths has probabilistic nature. The ratio between the two transition types was found to be sensitive to the elastic constants of LC forming the droplet. We suggest the principal approach for in situ estimation of ratios between elastic constants in cholesteric LCs deposited in polymer-dispersed LC material and discuss its strong and weak sides.
\end{abstract}

\section{Introduction}

The study of liquid crystals confined to microscopic cavities demonstrates growing interest for the last decades, both from the fundamental point of view ${ }^{1-5}$ and applications ${ }^{6-10}$, including sensors ${ }^{11-13}$, display technologies ${ }^{14}$, biological applications ${ }^{15,16}$ and other ${ }^{17-19}$. The direct connection between topological defects in particle physics and liquid crystals allows using liquid crystals as convenient systems for studies of several fundamental problems ${ }^{20-22}$. Last decade, the most intriguing discoveries shift from nematic to cholesteric liquid crystals (CLC). CLCs demonstrate plenty of various director configurations in confined geometries. For example, twisted bipolar structure ${ }^{13,23}$, structures with diametrical $\chi^{+1}$ or radial $\chi^{+2}$ dislocations ${ }^{23,24}$, uniform helix axis distribution ${ }^{25}$ and the double twisted the structure ${ }^{26}$ can be observed, as well as structures with the point defect in the bulk of CLC or at the surface and bipolar distribution of CLC axes ${ }^{27,28}$.

One of the practically important ways to fabricate, examine and use LCs in confined geometries are polymer-dispersed liquid crystal (PDLC) films. Typically, it is prepared by phase separation methods by mixing the polymer with LC in a homogeneous, single-phase blend. The subsequent phase separation can be induced in several ways. In temperature-induced phase separation (TIPS), a melted mixture of thermoplastic polymer and LC is placed on a substrate and gradually cooled down until polymer solidification induces phase separation (Fig. 1). In the end, LC becomes only partially soluble in a polymer matrix and form small LC droplets inside the film. While this approach has massive benefits like the ability to prepare material en masse and thermomechanical stability of the material, there are also payoffs. Most applications require using specific LC mixtures instead of pure LC compounds. Solubility rates of different parts of these compounds may significantly vary ${ }^{6,29-32}$. In turn, the content of an LC mixture in a PDLC droplet may differ from the original LC mixture due to various adsorption rates to the matrix of different components of a mix. And finally, it will affect the material properties of the LC mixture within the droplet (for example, elastic constants), its optical properties, and response to an electric field ${ }^{33,34}$. Thus it is essential to estimate the properties of LC material inside PDLC droplets after film preparation.
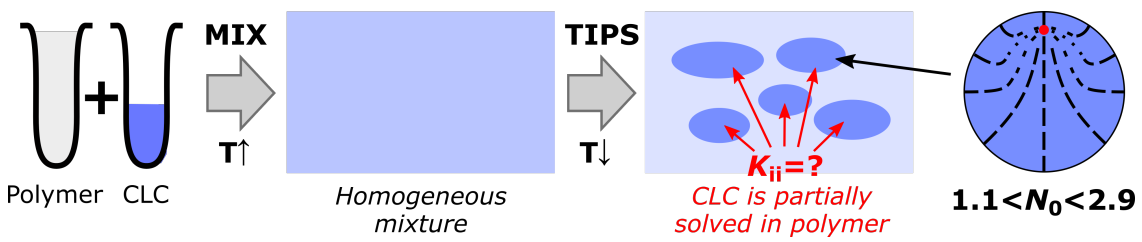

Figure 1. Principal scheme of preparation of polymer dispersed liquid crystalline material. After temperature-induced phase separation (TIPS), part of a liquid crystal remains solved in polymer, affecting the mixture contained in a droplet. 
There are seemingly two ways to solve the problem of estimating an LC mixture content in PDLC droplets. First, one can study adsorption rates and then evaluate the mixture composition. As there are many different LC compounds and polymer matrices, it is typically done theoretically ${ }^{35,36}$. Second, it is possible to measure optical structural features of the end droplets by means of microscopy and estimate material properties from it. This is an empirical approach requiring more complex studies. On the other side, its important benefit is that it can be based on experimental observations or directly compared with it. Recently this problem was addressed ${ }^{37}$ by simulating polarized microscopy images of a large set of structures for various elastic constants.

In this work, we studied the behavior of cholesteric droplets with homeotropic boundary conditions experimentally and by computer simulations. Small droplets forming twisted radial structures were studied. We obtained two different paths of structural transformations under electric fields in such droplets. The choice between these paths has probabilistic nature. The ratio between the two transition types was found to be sensitive to the elastic constants of LC forming the droplet. Thus we suggest the principal method for in situ estimation of ratios between elastic constants in cholesteric LCs deposited in PDLC material.

\section{Results}

PiBMA polymer assigns the homeotropic anchoring for the E7 nematic ${ }^{38}$. It is known that twisted radial defect (tR) structure with a single point defect shifted to the droplet edge is formed in the range of $N_{0}=2 d / p_{0}$ between 1.1 and 2.9 , where $d$ is droplet diameter and $p_{0}$ is equilibrium cholesteric pitch ${ }^{28}$. At $N_{0}=2.4(d=20 \mu \mathrm{m})$ this defect lies almost at the border (see Fig. 1 and Fig. 2, left column). Simultaneously, the high overall twist of the director field compensates for the twisting power of the CLC, which is visualized by the extinction lines of the radial structure. In simulations, this structure was fully confirmed in the range $1.3<N_{0}<3.2$.

\section{Transition dynamics under electric field}

We studied the behavior of droplets with $\mathbf{t R}$ structure under the action of an electric field. In both experiment and computer simulations, two possible sequences of structure transformation were obtained. In one case, the droplet remained axially symmetrical all the time; in another, the symmetry broke and was then restored only after transition to toron configuration. Below we depict both cases in detail.

Fig. 2 shows the sequence of symmetric droplet configurations in increasing electric field (hereafter the value of electric field is shown in dimensionless form $e=|\mathbf{E}| d\left(\varepsilon_{0} \Delta \varepsilon / 4 K_{11}\right)^{1 / 2}$, see details in Methods section). In Fig. 2 rows (a) and (b) we show experimental results for the droplets of $d=18 \mu \mathrm{m}\left(N_{0}=2.2\right)$ and $d=15 \mu \mathrm{m}\left(N_{0}=1.8\right)$, correspondingly. The structures in these droplets are very similar but oriented by $90^{\circ}$ to each other, as well as the electric field. The same transition in computer simulations is shown in rows (c)-(e) for a droplet with $N_{0}=2.5$. In all cases, in a low electric field, tR structure symmetry axis is parallel to the electric field, and point defect shifts close to the border of the droplet. In an intermediate electric field, the point defect slowly shifts toward the center of the droplet. Simultaneously, the twisted cone becomes thinner, which significantly changes its visual appearance in cross-polarized microscopy. Finally, the point defect breaks down into a circular line defect, and the droplet rapidly transforms into the toroidal configuration in a high electric field (Fig. 2, right column).

Another possible scenario is shown in Fig. 3. Here, the droplet has the same tR initial structure in the absence of an electric field (left column). However, at an intermediate electric field, the point defect shifts to the side, while the "base" of the twisting cone remains in the equatorial plane of the droplet. Thus, the droplet structure undergoes reorientation (Fig. 3, third column). Asymmetry becomes strongly pronounced, which is clearly seen in both cross-polarized microscopy images and director distribution. Finally, in a high electric field, the transition into toroidal configuration occurs (Fig. 3, fourth column). In the asymmetric scenario, it happens at a much lower $e$, than in symmetric case, regardless of droplet size.

\section{Probabilities of symmetric and asymmetric transitions}

In experiment and computer simulations, we obtained transitions through both scenarios in droplets of all sizes in the range of $N_{0}$ from $\sim 1.5$ to $\sim 3$. In the experiment, we were unable to determine any specific feature in a droplet at $e=0$, that would have determined the case. In simulations, a repeat of exactly the same state and simulation protocol led to one symmetric transition in some trials and asymmetric one in others. Thus the particular choice of transition scenario for every single droplet was seemingly probabilistic.

To quantify the experimental data, we analyzed transition type in 725 droplets with $N_{0}$ from $2.25 \pm 0.25$ to $3.75 \pm 0.25$. Only those droplets were taken into account, the symmetry axes of which were initially oriented mainly along the applied electric field. We considered the structural transition in a droplet as the symmetric scenario if the point defect shifted from the center less than half of the droplet radius in the whole range of $e$ before transition to the toroidal configuration. Otherwise, the case was counted as the asymmetric scenario. Then we split up all data by droplet size and calculated the probabilities of 


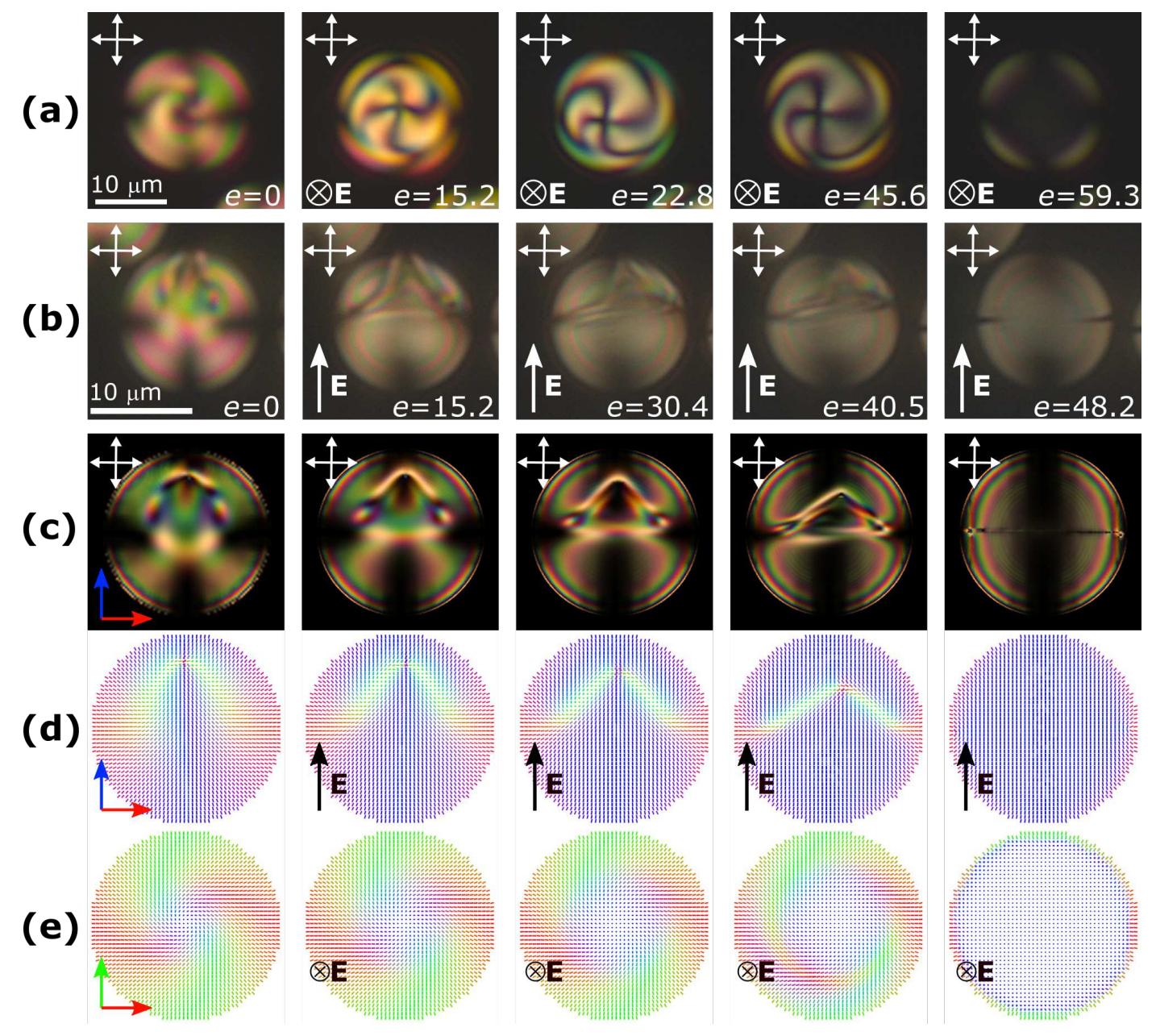

Figure 2. Sequence of structures for the symmetric transition scenario in increasing electric field $e=|\mathbf{E}| d\left(\varepsilon_{0} \Delta \varepsilon / 4 K_{11}\right)^{1 / 2}$. Experimental cross-polarized microscopy images for (a) symmetry axis perpendicular to the plane, $N_{0}=2.2$, and (b) symmetry axis parallel to the plane, $N_{0}=1.8$. Simulated cross-polarized images (c) and director distribution in primary cross cuts (d-e) for E7, $N_{0}=2.5$. Hereinafter, double arrows indicate the direction of the polarizers, and the director $\mathbf{n}$ is colored in correspondence with the direction (red along the $x$-axis, green along the $y$-axis, blue along the $z$-axis). 

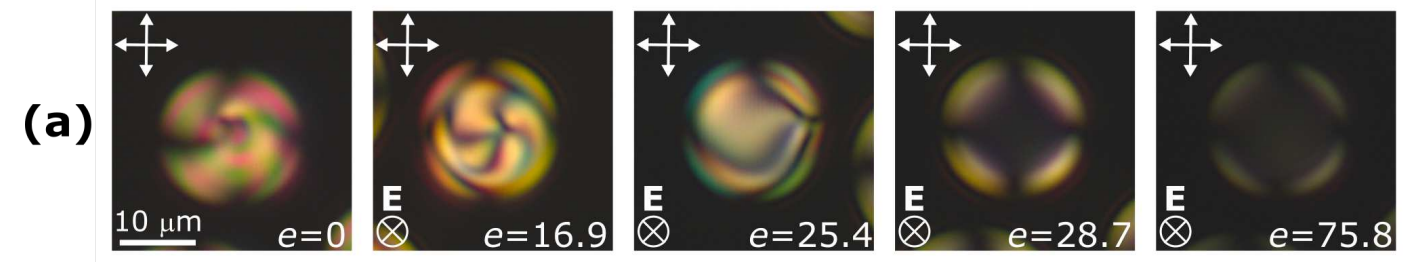

(b)
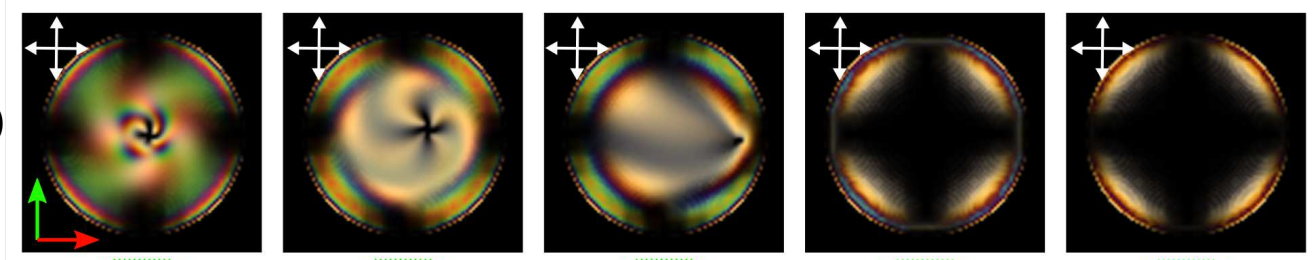

(c)
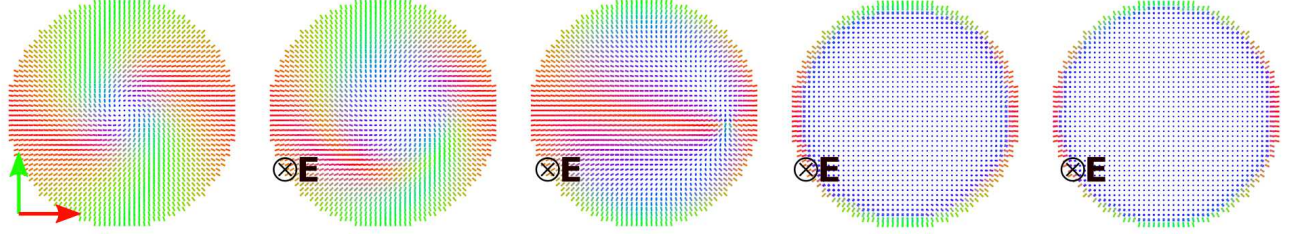

(d)
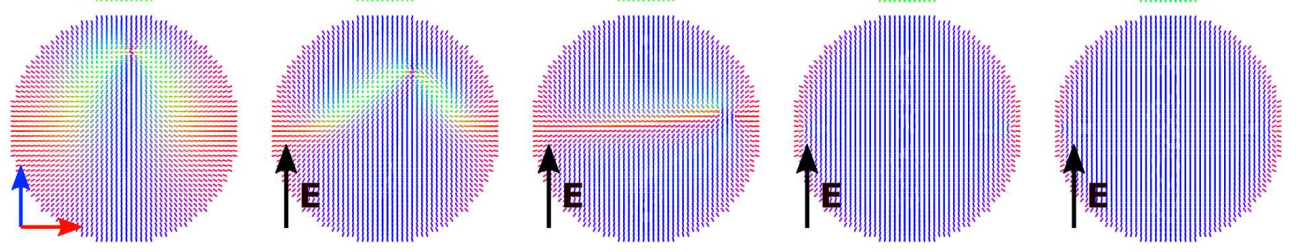

Figure 3. Sequence of structures for the asymmetric transition scenario in increasing electric field. Experimental cross-polarized microscopy images, $N_{0}=2.4$ (a), and simulated cross-polarized images (b) and director distribution in primary cross cuts (c,d) for $\mathrm{E} 7, N_{0}=2.5$. 
the two scenarios in each group (Fig. 4). On average, the probability of the asymmetric scenario was approximately $14 \%$ and showed only a slight dependency on droplet size.

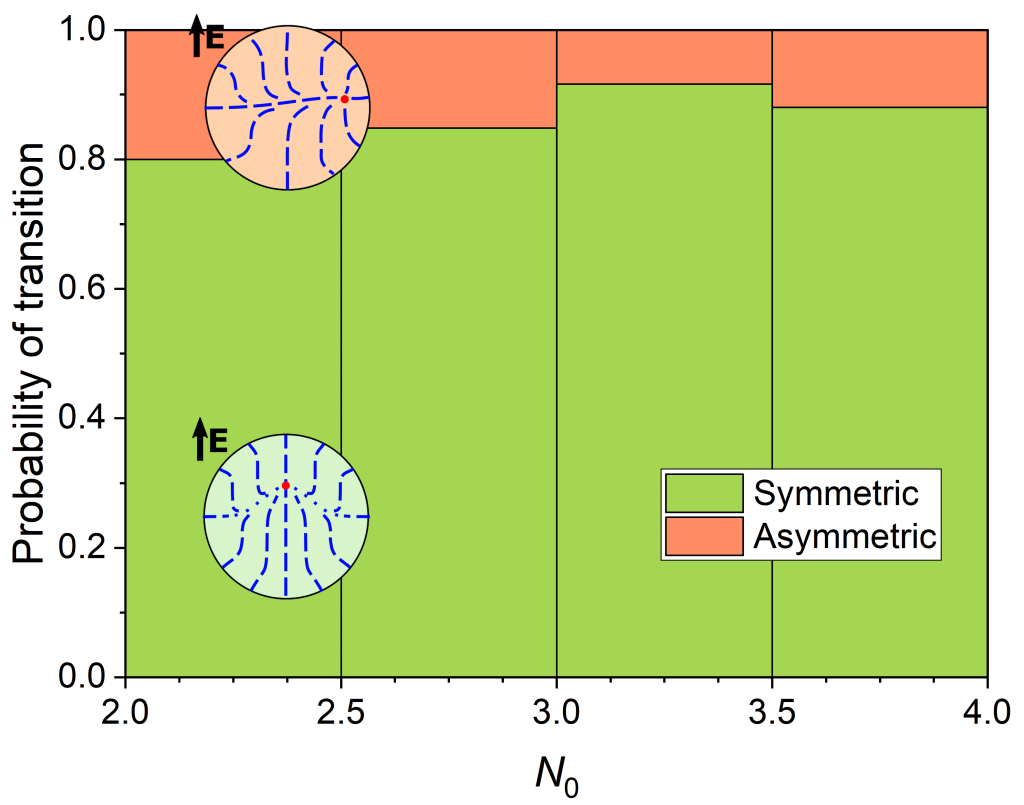

Figure 4. Probabilities of symmetric (green) and asymmetric (red) transitions at various relative droplet helicity parameters $N_{0}$, experimentally measured for 745 E7 droplets in PiBMA.

For theoretical analysis, we produced simulations of transition under the electric field for materials with various ratios between elastic constants $K_{22} / K_{11}$ and $K_{33} / K_{11}$. These ratios were changed simultaneously to obtain more realistic materials, including E7 and 5CB liquid crystals. In each case, we produced 150 independent simulations (total of 1350 runs) and split the results into three groups: symmetric, asymmetric, and unclear scenarios. The latter was assigned when the structure fluctuated significantly before the transition to toroidal configuration. Fig. 5 shows the resulting probability distribution. First, simulation results for E7 are very close to the experimental data. Second, the obtained distribution between probabilities of symmetric and asymmetric scenarios strongly depends on the elastic constants. Low $K_{22} / K_{11}$ appeared to be the most sensitive parts, with the number of symmetric cases rising from almost zero at $K_{22} / K_{11}=0.25$ to nearly two thirds for $5 \mathrm{CB}$. The changes between $5 \mathrm{CB}$ and E7 and further are less significant but still reasonably detectable.

\section{Conclusions and discussion}

We believe this effect potentially allows us to determine in situ the ratio between elastic constants in cholesteric LC mixtures inside the PDLC matrix. We consider the following blueprint of the protocol for such measurements: (1) estimate oblateness of the droplets under study; (2) estimate the possible range of values for the elastic constant ratios; (3) measure symmetric and asymmetric transition probabilities and check reference tables for the specific polymer (these data are to be collected in future studies).

We understand that the applied measurements require more detailed research and testing. First of all, more extensive studies in a broad range of elastic constant ratios are needed. Ideally, it should be done for pure LCs or LC mixtures with a known matrix absorption ratio. We understand it is very hard to implement for a single matrix type. Thus we suggest composing the complete reference datasets by combining experiments and simulations. Second, the influence of droplet oblateness on the transition probabilities may be also considered. While our approach utilizes small droplets $\left(N_{0}<3-4\right)$, which are almost spherical, some materials may result in oblate droplets. Previous data on droplet oblateness ${ }^{39}$ allows one to estimate it in a non-destructive manner. Third, it is unclear how to compare LC droplets in different matrixes. Different polymer materials may lead to varying strengths of surface anchoring, droplet shape, etc. Finally, the resulting numbers will strongly depend on the protocol in both experiments and simulations (for example, on the electric field growth rate). The data taken under different protocols can be compared only qualitatively but not quantitatively. An actual protocol can be adjusted to meet the technological requirements. As a primary option for laboratory measurements, we suggest the protocol used in this work. Despite these caveats, we believe the emphasized difficulties are vincible, as it requires more data but does not spoil the found effect. Thus we hope it can be used for in situ estimation of ratios of elastic constants in cholesteric LCs in the future. 


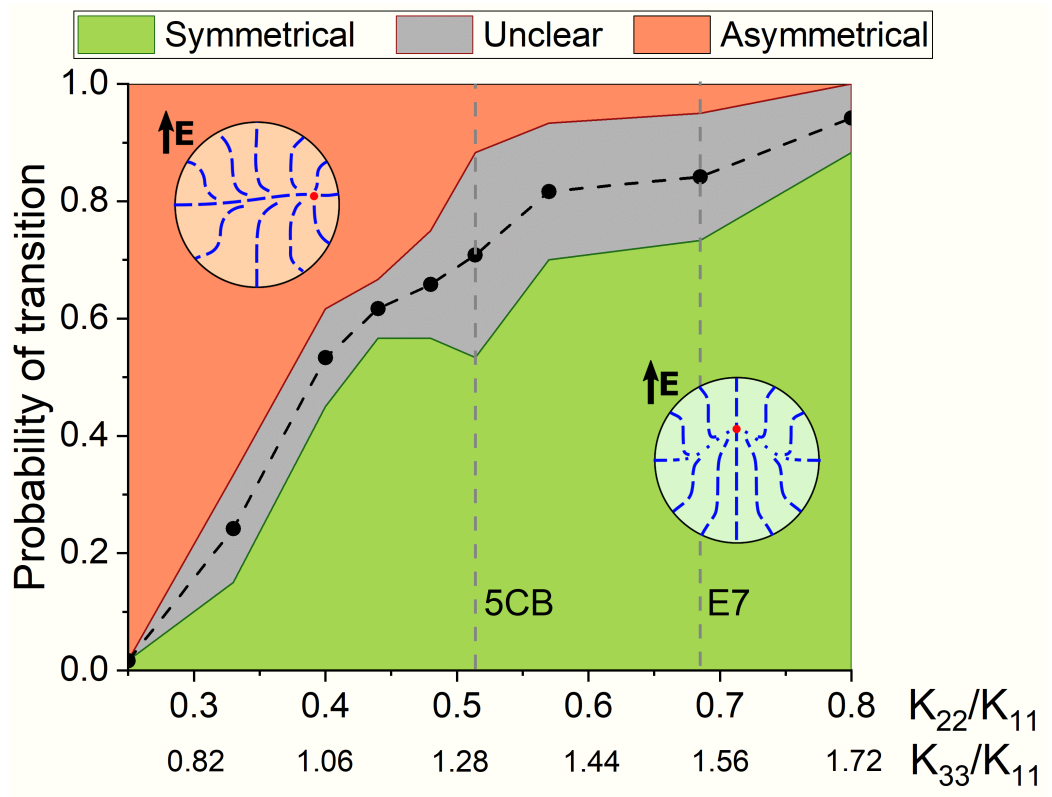

Figure 5. Probabilities of symmetric (green), asymmetric (red), and unclear (grey) transitions at relative droplet helicity parameter $N_{0}=2.5$ and various elastic constants, calculated from 150 computer simulation runs per point. The black dashed line shows the middle of the "unclear" zone, which estimates experimentally observable data.

\section{Methods}

\section{Experimental}

Experimental studies of the CLC droplet structures reaction were performed on polymeric dispersed liquid crystal (PDLC) films. PDLS films were based on poly(isobutyl methacrylate) (PiBMA) (Sigma Aldrich) and the nematic mixture E7 (Merck) doped cholesteryl acetate (Sigma Aldrich). The cholesteryl acetate concentration was $1 \mathrm{wt} \%$ corresponding to the intrinsic helix pitch $p_{0}=16.4 \mu \mathrm{m}$, was used to study the effect of the electric field on the cholesteric droplets structures. The concentration of cholesteryl acetate was $1.5 \mathrm{wt} . \%$, which corresponded to $p_{0}=10.9 \mu \mathrm{m}$ and was used to study the structural transitions statistical studies. The electro-optical cells were manufactured by combined phase separation technology techniques as described in ref. ${ }^{40}$ in detail. The manufacturing conditions were chosen such as to obtain droplets with a relative chiral parameter in the range $1.3<N_{0}<3.8$. Two types of electro-optical cells were used, in which the electric field was applied i) perpendicularly or ii) along the plane of the PDLC film. Two identical substrates with transparent ITO electrodes on the inner sides were used to make electro-optical cells of the first type. The investigated PDLC film was placed between the substrates. Electro-optical cells of the second type, the electrodes were located only on the lower substrate at a distance approximately equal to $H \cong 100 \mu \mathrm{m}$ from each other. The top substrate was used without electrodes since its function is to set the PDLC film thickness. The composite film thickness was specified by $20 \mathrm{~m}$ diameter glass microspheres (Duke Scientific Corporation). Experimental studies were carried out using a polarizing optical microscope (POM) Axio Imager.A1m (Carl Zeiss). AC electric field with a frequency of 1 $\mathrm{kHz}$ was applied to the electro-optical cells. The voltage amplitude was increased step by step with a step of $1 \mathrm{~V}$ RMS (for both types of electro-optical cell) every $60 \mathrm{~s}$.

\section{Computer simulations}

\section{Structure calculations}

We performed simulations of the LC structure within spherical droplets with homeotropic boundaries filled with chiral nematic. The volume was rendered in $48 \times 48 \times 48$ lattices. We used extended Frank elastic continuum approach with Monte-Carlo annealing optimization ${ }^{41}$ to find the energy-optimal droplet structures. This approach includes the effects of the director field distortion and the formation of defects in the droplet:

$$
\begin{aligned}
F=\int_{V}\left(\frac{K_{11}}{2}(\operatorname{divn})^{2}+\frac{K_{22}}{2}\left(\mathbf{n} \cdot \operatorname{rotn}+q_{0}\right)^{2}+\frac{K_{33}}{2}[\mathbf{n} \times\right. & \left.\operatorname{rotn}]^{2}\right) d V+\frac{W}{2} \int_{\Omega}\left(1-\cos ^{2} \gamma\right) d \Omega \\
& +F_{\text {def }}+\varepsilon_{0} \Delta \varepsilon \int_{V}\left(\mathbf{E}^{2}-(\mathbf{E} \cdot \mathbf{n})^{2}\right) d V
\end{aligned}
$$


where $K_{11}, K_{22}$ and $K_{33}$ are the splay, twist and bend elasticity constants, respectively, $q_{0}=2 \pi / p_{0}, W$ is the surface anchoring energy density, $\gamma$ is the angle between local director and normal to the droplet surface, $F_{\text {def }}$ is the energy of defects calculated by the summation of the point and linear defect energies, and $\Delta \varepsilon$ is dielectric anisotropy of LC, $\mathbf{E}$ is the electric field. The types, positions and energies of defects were estimated automatically during the Monte-Carlo optimization procedure (see the details in ref. ${ }^{41}$ ).

The initial structures in absence of an electric field, including the director field, the types, positions, and energies of defects were calculated during the Monte-Carlo optimization procedure (see the details in ref. ${ }^{41}$ ). To take into account potential formation of the disclination lines with core, its linear energy density was set to $f_{\text {core }}^{\text {line }}=2.75 K_{11}$ ( same as in $^{28}$ ). The relative chiral parameter $N_{0}=2 d / p_{0}$ has been varied from 2.0 to 3.0. The homeotropic anchoring strength was set to strong, $\mu=W d / 2 K_{11}$ varied from 1500 (for $N_{0}=2.7$ ) to 2400 (for $N_{0}=4.3$ ) to maintain constant natural units value of $W=1.75 \times 10^{-3} \mathrm{~J} / \mathrm{m}^{2}$.

The ratio between elasticity constants was set to $K_{11}: K_{22}: K_{33}=1: 0.685: 1.54$ to simulate the cholesteric liquid crystal mixture under study. The relative chiral parameter $N_{0}$ has been varied from 2.7 to 4.3 . The homeotropic anchoring strength was set to strong, $\mu=W d / 2 K_{11}$ varied from 1500 (for $N_{0}=2.7$ ) to 2400 (for $N_{0}=4.3$ ) to maintain constant natural units value of $W=1.75 \times 10^{-3} \mathrm{~J} / \mathrm{m}^{2}$. To simulate changes in the structure under a slowly increasing electric field, we increased the the amplitude of the electric field in small steps $(\Delta e=0.25)$ and applied Monte Carlo relaxation at each step $\left(10^{5}\right.$ Monte Carlo multisteps). The data are shown in dimensionless electric field, which were calculated as $e=|\mathbf{E}| d\left(\varepsilon_{0} \Delta \varepsilon / 4 K_{11}\right)^{1 / 2}$.

\section{Calculation of the POM textures}

We have calculated the POM textures of spherical-cap droplets using the Jones matrix technique, formulated for PDLC materials in ref. ${ }^{42}$. This technique supposes direct unidirectional propagation of linearly polarized light through a non-uniform birefringent material. Light diffraction, diffusion, and scattering are not taken into account in the Jones calculus, and thus textures on the peripheral parts of the droplets are roughly estimated. Textures were calculated for ten different wavelengths within the visible spectrum, from $400 \mathrm{~nm}$ to $700 \mathrm{~nm}$ with the equal step of $33 \mathrm{~nm}$. The values of ordinary and extraordinary reflective indices for $\mathrm{E} 7$ cholesteric were set in according to ${ }^{43}$. Color textures were created by merging the individual wavelength textures with regard to the luminescence spectra of the black body at a temperature $\approx 3000 \mathrm{~K}$.

\section{References}

1. Pinkevich, I. P., Reshetnyak, V. Y., Rezinkov, Y. A. \& Smirnov, A. G. Influence of anchoring energy on orientational ordering and phase transition in nematic droplets in polymer matrix. Mol. Cryst. Liq. Cryst. Sci. Technol. Sect. A. Mol. Cryst. Liq. Cryst. 222, 279-286, DOI: 10.1080/15421409208048702 (1992).

2. Kleman, M. \& Lavrentovich, O. D. Topological point defects in nematic liquid crystals. Philos. Mag. 86, 4117-4137, DOI: 10.1080/14786430600593016 (2006).

3. Seč, D., Porenta, T., Ravnik, M. \& Žumer, S. Geometrical frustration of chiral ordering in cholesteric droplets. Soft Matter 8, 11982, DOI: $10.1039 / \mathrm{c} 2 \mathrm{sm} 27048 \mathrm{j}$ (2012).

4. Alexander, G. P., ge Chen, B. G., Matsumoto, E. A. \& Kamien, R. D. Colloquium: Disclination loops, point defects, and all that in nematic liquid crystals. Rev. Mod. Phys. 84, 497-514, DOI: 10.1103/revmodphys.84.497 (2012).

5. Čopar, S., Ravnik, M. \& Žumer, S. Janus nematic colloids with designable valence. Materials 7, 4272-4281, DOI: 10.3390/ma7064272 (2014).

6. Drzaic, P. S. Liquid Crystal Dispersions (World Scientific, 1995).

7. Humar, M. \& Muševič, I. 3d microlasers from self-assembled cholesteric liquid-crystal microdroplets. Opt. Express 18, 26995, DOI: 10.1364/oe.18.026995 (2010).

8. Nikkhou, M. et al. Light-controlled topological charge in a nematic liquid crystal. Nat. Phys. 11, 183-187, DOI: 10.1038 /nphys3194 (2014).

9. Humar, M., Araoka, F., Takezoe, H. \& Muševič, I. Lasing properties of polymerized chiral nematic bragg onion microlasers. Opt. Express 24, 19237, DOI: 10.1364/oe.24.019237 (2016).

10. Zhang, Y.-S. et al. Thermal and optical manipulation of morphology in cholesteric liquid crystal microdroplets constrained on microfibers. J. Mol. Liq. 328, 115383, DOI: 10.1016/j.molliq.2021.115383 (2021).

11. Woltman, S. J., Jay, G. D. \& Crawford, G. P. Liquid-crystal materials find a new order in biomedical applications. Nat. Mater. 6, 929-938, DOI: 10.1038/nmat2010 (2007). 
12. Lee, H.-G., Munir, S. \& Park, S.-Y. Cholesteric liquid crystal droplets for biosensors. ACS Appl. Mater. \& Interfaces 8 , 26407-26417, DOI: 10.1021/acsami.6b09624 (2016).

13. Zhou, Y. et al. Structural transitions in cholesteric liquid crystal droplets. ACS Nano 10, 6484-6490, DOI: 10.1021/ acsnano.6b01088 (2016).

14. Ahoff, S. J. et al. Superstructures of chiral nematic microspheres as all-optical switchable distributors of light. Sci. Reports 5, DOI: $10 . \overline{1038} / \mathrm{srep} 14183$ (2015).

15. Beller, D. A. et al. Geometry of the cholesteric phase. Phys. Rev. X 4, DOI: 10.1103/physrevx.4.031050 (2014).

16. Tran, L. et al. Change in stripes for cholesteric shells via anchoring in moderation. Phys. Rev. X 7, DOI: 10.1103/physrevx. 7.041029(2017).

17. Wang, Y. et al. Tunable whispering gallery modes lasing in dye-doped cholesteric liquid crystal microdroplets. Appl. Phys. Lett. 109, 231906, DOI: 10.1063/1.4971973 (2016).

18. Belmonte, A., Bus, T., Broer, D. J. \& Schenning, A. P. Patterned full-color reflective coatings based on photonic cholesteric liquid-crystalline particles. ACS Appl. Mater. \& Interfaces 11, 14376-14382, DOI: 10.1021/acsami.9b02680 (2019).

19. Chen, C., Kim, D. \& Jhun, C. Electro-optical effects of a color polymer-dispersed liquid crystal device by microencapsulation with a pigment-doped shell. Crystals 9, 364, DOI: 10.3390/cryst9070364 (2019).

20. Smalyukh, I. I., Lansac, Y., Clark, N. A. \& Trivedi, R. P. Three-dimensional structure and multistable optical switching of triple-twisted particle-like excitations in anisotropic fluids. Nat. Mater. 9, 139-145, DOI: 10.1038/nmat2592 (2009).

21. Kurioz, P., Kralj, M., Murray, B. S., Rosenblatt, C. \& Kralj, S. Nematic topological defects positionally controlled by geometry and external fields. Beilstein J. Nanotechnol. 9, 109-118, DOI: 10.3762/bjnano.9.13 (2018).

22. Ackerman, P. J. \& Smalyukh, I. I. Diversity of knot solitons in liquid crystals manifested by linking of preimages in torons and hopfions. Phys. Rev. X 7, DOI: 10.1103/physrevx.7.011006 (2017).

23. Bezić, J. \& Žumer, S. Structures of the cholesteric liquid crystal droplets with parallel surface anchoring. Liq. Cryst. 11, 593-619, DOI: 10.1080/02678299208029013 (1992).

24. Darmon, A., Benzaquen, M., Čopar, S., Dauchot, O. \& Lopez-Leon, T. Topological defects in cholesteric liquid crystal shells. Soft Matter 12, 9280-9288, DOI: 10.1039/c6sm01748g (2016).

25. Bouligand, Y. \& Livolant, F. The organization of cholesteric spherulites. J. de Physique 45, 1899-1923, DOI: 10.1051/jphys: 0198400450120189900 (1984).

26. Yoshioka, J., Ito, F. \& Tabe, Y. Stability of a double twisted structure in spherical cholesteric droplets. Soft Matter 12, 2400-2407, DOI: 10.1039/c5sm02838h (2016).

27. Krakhalev, M. N. et al. Bipolar configuration with twisted loop defect in chiral nematic droplets under homeotropic surface anchoring. Sci. Reports 7, DOI: 10.1038/s41598-017-15049-6 (2017).

28. Krakhalev, M. N. et al. Orientational structures in cholesteric droplets with homeotropic surface anchoring. Soft Matter 15, 5554-5561, DOI: 10.1039/c9sm00384c (2019).

29. Smith, G. W. \& Vaz, N. A. Methods for Determination of Solubility Limits of Polymer-Dispersed Liquid Crystals. Mol. Cryst. Liq. Cryst. Sci. Technol. Sect. A. Mol. Cryst. Liq. Cryst. 237, 243-269, DOI: 10.1080/10587259308030140 (1993).

30. Smith, G. W. \& Vaz, N. A. The relationship between formation kinetics and microdroplet size of epoxy-based polymerdispersed liquid crystals. Liq. Cryst. 3, 543-571, DOI: 10.1080/02678298808086401 (1988).

31. Rout, D. K. \& Jain, S. C. Dielectric Properties of a Polymer-Dispersed Liquid Crystal Film. Mol. Cryst. Liq. Cryst. Sci. Technol. Sect. A. Mol. Cryst. Liq. Cryst. 210, 75-81, DOI: 10.1080/10587259208030758 (1992).

32. Reyes, C. G., Baller, J., Araki, T. \& Lagerwall, J. P. F. Isotropic-isotropic phase separation and spinodal decomposition in liquid crystal-solvent mixtures. Soft Matter 15, 6044-6054, DOI: 10.1039/C9SM00921C (2019).

33. Chandrasekhar, S. Liquid Crystals (Cambridge University Press, 1992).

34. de Gennes, P. G. \& Prost, J. The Physics of Liquid Crystals (Oxford University Press, 1993).

35. Xu, J., Liu, H., Li, W., Zou, H. \& Xu, W. Application of QSPR to binary polymer/solvent mixtures: Prediction of flory-huggins parameters. Macromol. Theory Simulations 17, 470-477, DOI: 10.1002/mats.200800063 (2008). 
36. Erlebach, A. et al. Predicting solubility of small molecules in macromolecular compounds for nanomedicine application from atomistic simulations. Adv. Theory Simulations 3, 2000001, DOI: 10.1002/adts.202000001 (2020).

37. Chiccoli, C., Pasini, P. \& Zannoni, C. Can elastic constants and surface alignment be obtained from polarized microscopy images of nematic droplets? a monte carlo study. J. Mol. Liq. 267, 158-165, DOI: 10.1016/j.molliq.2017.12.045 (2018).

38. Xie, A. \& Higgins, D. A. Electric-field-induced dynamics in radial liquid crystal droplets studied by multiphoton-excited fluorescence microscopy. Appl. Phys. Lett. 84, 4014-4016, DOI: 10.1063/1.1748846 (2004).

39. Gardymova, A. P. et al. Polymer dispersed cholesteric liquid crystals with a toroidal director configuration under an electric field. Polymers 13, 732, DOI: 10.3390/polym13050732 (2021).

40. Gardymova, A. P. et al. Polymer dispersed cholesteric liquid crystals with a toroidal director configuration under an electric field. Polymers 13, 732, DOI: 10.3390/polym13050732 (2021).

41. Rudyak, V. Y., Emelyanenko, A. V. \& Loiko, V. A. Structure transitions in oblate nematic droplets. Phys. Rev. E 88 (2013).

42. Ondris-Crawford, R. et al. Microscope textures of nematic droplets in polymer dispersed liquid crystals. J. Appl. Phys. 69, 6380-6386 (1991).

43. Li, J., Wen, C.-H., Gauza, S., Lu, R. \& Wu, S.-T. Refractive indices of liquid crystals for display applications. J. Disp. Technol. 1, 51 (2005).

\section{Acknowledgements}

This research was funded by Russian Science Foundation, Grant No. 20-72-10038. The research was carried out using the equipment of the shared research facilities of HPC computing resources at Lomonosov Moscow State University.

\section{Author contributions statement}

V.Yu.R. initiated this study, A.P.G. fabricated the PDLC cells, M.N.K. and A.P.G. conducted the experimental studies of the orientational transformations in CLC droplets, A.S.A. carried out an experimental study of realization probability of the transitions scenario, V.Yu.R. carried out computer simulations, formal analysis, methodology, software, visualization, A.A.A. carried out computer simulations of realization probability of the transitions scenario, V.Yu.R. and V.Ya.Z. supervised the study. All authors reviewed the manuscript.

\section{Competing interests}

The authors declare no competing interests.

\section{Data availability}

The datasets generated during and analysed during the current study are available from the corresponding author on reasonable request. 